\title{
Theoretical Investigation of the Electronic, Elastic, Vibration, Thermodynamic and Transport Properties of PtAsP Mixed Pyrite Phase
}

\author{
Adewumi I. Popoola and B. Samuel Agboola
}

\section{ABSTRACT}

Theoretical investigation on the elastic constants, phonon frequencies, thermodynamic and the transport properties of PtAsP mixed cubic pyrite phase was performed using the first-principles calculations based on the density functional theory. The calculated equilibrium crystal parameter is in excellent agreement with experimental data. The derived bulk and shear moduli are much higher than other theoretical data, suggesting that PtAsP may be a highly incompressible material. The detailed analyses of the electronic structures showed that PtAsP is an indirect energy gap compound and is elastically and dynamically stable. By using the harmonic Debye model, some thermodynamic properties including vibration free energy and constant volume heat capacity were calculated. The evaluation of the transport properties showed that PtAsP is a p-type material with capacity for improved performance when its charge carrier concentration is between $10^{16} \mathrm{~cm}^{3}$ and $10^{18} \mathrm{~cm}^{3}$.

Keywords: DFT, Seebeck Coefficient, Phonon, Density of State.
Published Online: January 4, 2021

ISSN: $2684-4451$

DOI :10.24018/ejphysics.2021.3.1.40

\section{A. I. Popoola*}

Department of Physics,

Federal University of Technology,

Akure, Ondo State, Nigeria.

(e-mail: aipopoola@futa.edu.ng)

B. S. Agboola

Department of Physics,

Federal University of Technology,

Akure, Ondo State, Nigeria.

(e-mail: bsagboola@futa.edu.ng)

*Corresponding Author

\section{INTRODUCTION}

A large number of transition element compounds of the form $Y X_{2}$ crystallizes in the pyrite structure (prototypes includes $\mathrm{FeS}_{2}, \mathrm{PdAs}_{2}, \mathrm{PtAs}_{2}, \mathrm{PtP}_{2} \& \mathrm{PtBi}_{2}$ ). This group have attracted intense research efforts, due to their varied but remarkable properties [1]-[3]. Mixed pyrite phases such as PtAsP, PtSbBi and PdAsSb have also been identified [4]. The crystal structure of cubic pyrite and that of the mixed cubic pyrite phase are shown in Fig. 1. While extensive investigation has been carried out by several researchers on the cubic pyrites, little information is available on the mixed cubic pyrites. The bandgap of PtAsP have been predicted to be $0.815 \mathrm{eV}$ while PdAsSb and PtSbBi have zero bandgaps [5]. For photonic, optoelectronic, and thermoelectric applications, semiconductors are the material of choice. A search of available material databases only yielded optimized structural data on PtAsP without detailed band structures, full elastic tensor, and other properties of value. Moreover, the electronic structures, elastic stiffness, thermodynamic as well as the transport properties deserve attention so as to extend our knowledge about the material's performance. The influence of elastic properties on the elastic deformation behavior and the possible role of anisotropy of interatomic bonding are for example, important to many practical applications. With these data lacking both in experiment and theoretically, it is the aim of this research to investigate the electronic, elastic, vibration, thermodynamic and the transport properties of the PtAsP mixed pyrite phase. The explicit determination of these fundamental properties is of particular interest due to their impact on electronic devices. Specifically, data on the cubic elastic constants, the Young modulus, shear modulus, elastic wave velocities, Poisson ratio, elastic anisotropy, Kleinman parameter, anisotropy degree are still lacking for PtAsP. The interest of the research is explored through firstprinciples methods for geometry optimization and phonon calculation effects. The quasi-harmonic Debye model is used to provide information on the thermodynamic properties. To our understanding, this is the first detailed investigation reporting on these parameters. The rest of the paper is as follows. The method adopted for the calculations are highlighted in Section 2. Our calculations results are presented and discussed in Section 3. We then summarize and conclude in Section 4.

\section{Computation Methods}

For a molecule or periodic systems, geometry optimization is required to get a stable configuration in DFT calculations. This is achieved by sampling repeatedly, the potential energy surface until a minimum (i.e., forces on all atoms are zero) is attained. The calculations leading to the results reported in this article were performed using the pseudo potential plane wave method implemented in the Quantum Espresso computer code [6]. The Perdew-BurkeErnzerhof form of the generalized gradient approximations (GGA) [7] was used to treat the electron exchangecorrelation potential. Optimized results were obtained at first Brillouin-zone integration approximated by $1000 \mathrm{k}$ points $(10 \times 10 \times 10)$ according to the Monkhorst-Pack [8] scheme. For self-consistent calculation, the energy convergence criterion was $10^{-4}$ Ry. After relaxing the unit cell volume, the ground state optimized lattice constant was 
calculated using the Murnaghan's equation of state [9]. To evaluate the lattice dynamics, phonon frequencies have been calculated as a second-order derivative of the total energy with respect to atomic displacements within the frame work of the Density Functional Perturbation Theory (DFPT) [10]. The calculation of the transport properties such as the Seebeck coefficient, electrical conductivity, and electronic thermal conductivity have been carried out using BoltzTrap-1.2.5, a computer code that solves the Boltzmann transport equation under the constant relaxation time $(\tau)$ approximation [11].

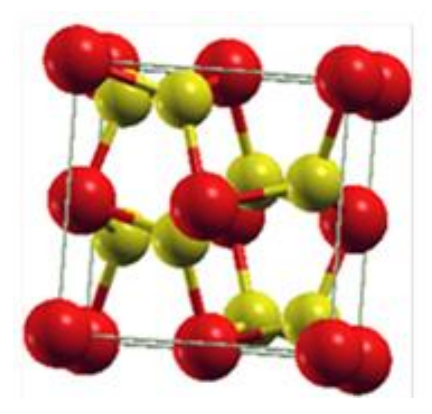

(a)

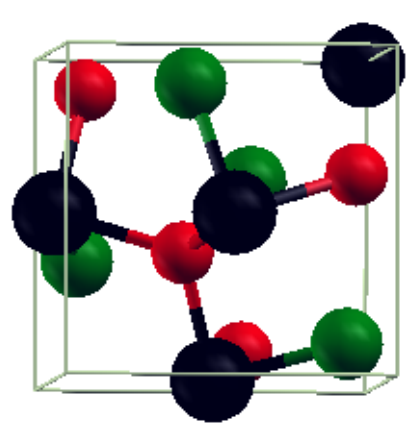

(b)
Fig. 1. (a) crystal structure for binary cubic pyrite [space group $=\mathrm{Pa}-3$ (no 205)],

(b) crystal structure for mixed cubic pyrite [space group $=\mathrm{P} 2{ }_{1} 3$ (no. 198)]

\section{RESULTS AND DISCUSSION}

\section{A. Elastic Properties}

There are three elastic constants $\left(\mathrm{C}_{11}, \mathrm{C}_{12}\right.$ and $\left.\mathrm{C}_{44}\right)$ needed to sufficiently specify the stability of PtAsP. These constants, which can be derived from the total energy/stress calculations, represent the single crystal elastic constants. A more convenient approach for obtaining the elastic constants of polycrystalline materials uses the Voigt-ReussHill approximation [12]. To obtain more reliable elastic constants and other connected parameters we have used the Voigt-Reuss-Hill approximated values. The obtained elastic constants together with connected parameters alongside experimental as well as theoretical data from other sources are listed in Table 1. For a cubic structure to be elastically stable it must satisfy the Born structural stability criteria [13] given as: $\mathrm{C}_{11}-\mathrm{C}_{12}>0, \mathrm{C}_{11}>0, \mathrm{C}_{44}>0, \mathrm{C}_{11}+2 \mathrm{C}_{12}>0$ and $\mathrm{C}_{12}<B<\mathrm{C}_{11}$. A quick look at Table 1 shows that all the conditions are satisfied by PtAsP. Thus, the present $C_{11}, C_{12}$ and $\mathrm{C}_{44}$ values adequately support both structural and cubic stability conditions for PtAsP.

The bulk modulus $(B)$, specifies the amount of resistance a material offers to external deformation. It also relates to the bonding strength of a material [14], [15]. The shear modulus $(G)$ on the other hand, refers to the resistance to shape change created by a shearing force [16], [17]. The resistance created against uniaxial tensions is known as the Young modulus $(E)$. $E$ measures the degree of stiffness of a material (i.e., the higher $E$ is, the stiffer the material) [18], [19]. The compared numerical $B$ and $G$ in Table 1 show that the data from this present calculation are substantially higher than the one from a previous calculation [5].
TABLE I: CALCULATED ISOTROPIC BULK MODULUS $B$, SHEAR MODULUS $G$, YounG's modulus $E$, Pugh RATIO $(B / G)$, ElastiC CONSTANTS $\left(\mathrm{C}_{11}, \mathrm{C}_{12}\right.$, $\mathrm{C}_{44}$ ), ZENER ANISOTROPY FACTOR $A$, KLEINMAN PARAMETER Z, POISSON'S RATIO N, SOUND VELOCITIES $\left(V_{T}, V_{L}, V_{\mathrm{M}}\right)$, THE DEBYE TEMPERATURE $\Theta_{\mathrm{D}}$, THE DENSITY P, THE LATTICE PARAMETER AND BAND-GAP SIZE FOR PTASP.

\begin{tabular}{cc} 
RESULTS FROM OTHER SOURCES ARE IN BRACKETS \\
\hline Properties & PtAsP \\
\hline$B(\mathrm{GPa})$ & $172.89[112.79]^{*}$ \\
$G(\mathrm{GPa})$ & $101.99[45.23]^{*}$ \\
$B / G$ & 1.70 \\
$E(\mathrm{GPa})$ & 255.68 \\
$\mathrm{C}_{11}(\mathrm{GPa})$ & 363.39 \\
$\mathrm{C}_{12}(\mathrm{GPa})$ & 77.64 \\
$\mathrm{C}_{44}(\mathrm{GPa})$ & 81.17 \\
$A$ & 0.57 \\
$\zeta$ & 0.36 \\
$v$ & 0.25 \\
$C_{\mathrm{p}}(\mathrm{GPa})$ & -3.53 \\
$v_{t}(\mathrm{~m} / \mathrm{s})$ & 3317 \\
$v_{l}(\mathrm{~m} / \mathrm{s})$ & 5772 \\
$v_{m}(\mathrm{~m} / \mathrm{s})$ & 3654 \\
$\Theta_{\mathrm{D}}(\mathrm{K})$ & 455.82 \\
$\rho\left(\mathrm{g} / \mathrm{cm}{ }^{3}\right)$ & {$[9.682]$} \\
$a(\AA)$ & {$[0.815]^{*}$} \\
$E_{\mathrm{g}}(\mathrm{eV})$ & 0.038 \\
$A^{*}$ & $5.849[5.911]^{*}[5.842]^{* *}$ \\
\hline
\end{tabular}

In engineering, the ductility or brittleness of materials play key roles. Brittle materials are generally not malleable or less deformable before fracture. Conversely, ductile materials are malleable and thus can accommodate a lot of deformation before fracture. One of the ways to theoretically appraise the ductile (brittle) nature of a material at ambient pressure is using the Pugh ratio. A material is considered to be ductile if its Pugh ratio $(B / G)$ is about 1.75 and higher, otherwise the material is brittle [20]. The Cauchy pressure $C_{\mathrm{p}}$ [defined as: $C_{\mathrm{p}}=\mathrm{C}_{12}-\mathrm{C}_{44}$ ] is also a reliable and an alternative way of gauging the ductility/brittleness of a material. A negative $C_{\mathrm{p}}$ stands for brittleness; otherwise, the material is ductile [21]. According to the data in Table 1, PtAsP is predicted to be brittle $\left(B / \mathrm{G}<1.75 \& C_{\mathrm{p}}<0\right)$ at ambient condition. Bonding forces/strength is a factor that also characterizes a material. The Poisson ratio ( $v$ ) relates the transverse and the longitudinal strains along the elastic loading directions. It can thus be theoretically used to gauge bond types in a material. A material with $v \approx 0.1$ is considered to be covalently bonded, those with $v \approx 0.25$ are ionic and for metallic bonds, $v \approx 0.33$ [22] - [24]. Based on this norm, it can be predicted that the dominant bond in PtAsP is ionic $(v$ $=0.25)$. In solids under ambient conditions, vibrational excitations are responsible for two typical elastic waves longitudinal waves $\left(v_{l}\right)$ and transverse $\left(v_{t}\right)$ waves [23]. The predicted values for these waves and their average $\left(v_{m}\right)$ are listed in Table 1. The ease of bond bending to bond stretching is predicted by the Kleinman parameter $(\zeta)$. When bond bending is minized, $\zeta=0$. When bond stretching is minimized, $\zeta=1$. The Kleinman parameter [25], links to the elastic constants as: $\zeta=\mathrm{C}_{11}+8 \mathrm{C}_{12} / 7 \mathrm{C}_{11}+$ $2 \mathrm{C}_{12}$. In PtAsP, bond bending is much lower compared to bond stretching.

\section{B. Electronic and Vibration Properties}

Many physical and chemical properties of solids correlate strongly with the electronic structure. The calculated electronic structure (density of states and band-structure) 
for PtAsP is presented in Figure 2(a). It is clear from Fig. 2 (a) that PtAsP would exhibit indirect energy band-gap. The band character seen between the X and GAMMA $(\Gamma)$ point along the Brillouin zones where a band spills to the Fermi level (Fermi level indicated by red line on the plots) should predispose PtAsP towards high electronic carrier mobility. On the Density of States (DoS) plots (Fig. 2 (a)-right), one can see that the spectrum with highest DoS are on the nonbonding side of the plot. This predicts that PtAsP should be structurally stable. To investigate the stability of PtAsP due to phonon vibrations, its phonon dispersion along several lines through the first Brillouin zone of the primitive unit cell (Fig. 2 (b)) and the phonon density of states (Fig. 2 (c)) at zero pressure have been calculated. The absence of phonons of negative frequency in Fig. 2 (b) shows that PtAsP is dynamically stable. There is no experimental data on phonon frequencies for PtAsP for comparison. The phonon density of states for PtAsP (Fig.2 (c)), shows that the peaks from 125 to $135 \mathrm{~cm}^{-1}$ correspond to the low frequency acoustic modes associated with bond bending. The optical mode transverse high frequency can be predicted to be around $277 \mathrm{~cm}^{-1}$.

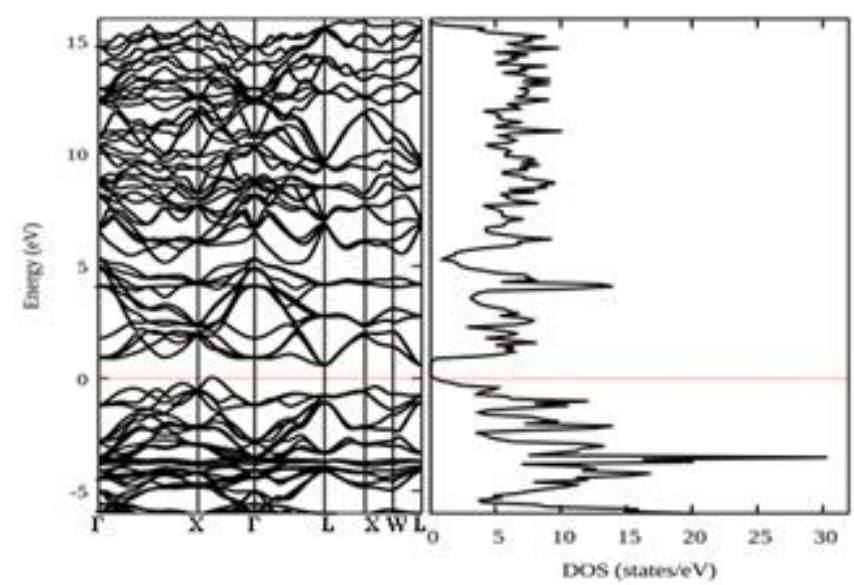

(a)
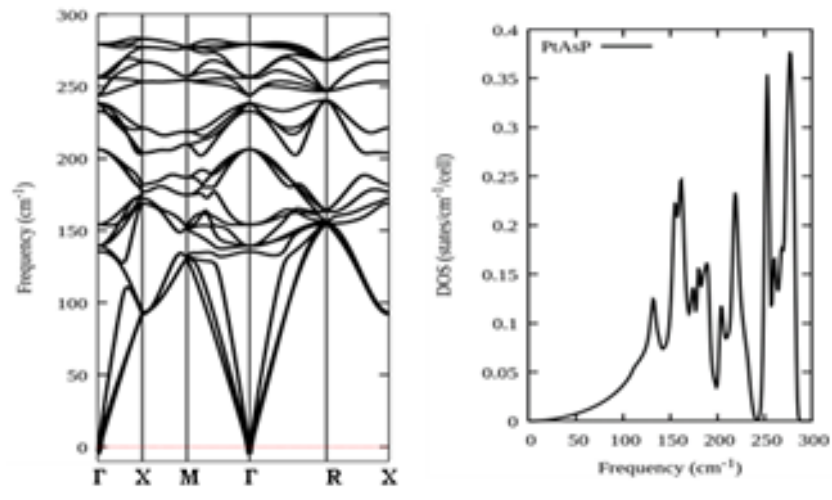

(b)

Fig. 2. Calculated electronic band structure and density of states (a), phonon dispersion (b), and the phonon density of states for PtAsP.

\section{Thermodynamic Properties}

Because of the behaviour of materials at elevated temperatures, it is almost always an interesting effort to investigate the temperature dependent thermal properties. After obtaining the phonon density of states, the thermal properties of PtAsP in the harmonic approximation were found. The graphs in Fig. 3, shows the vibrational free energy, the entropy, the total vibrational energy, and the heat capacity at ambient pressure. As expected, the total vibration energy (Fig. 3 (a)), is seen to have linear relation with temperature from room temperature to $800 \mathrm{~K}$. The vibration free energy relates to the thermal stability of any material. For PtAsP, the vibration free energy is $19.67 \mathrm{~kJ} / \mathrm{K} /(\mathrm{N} \mathrm{mol})$ at $300 \mathrm{~K}$ and goes negative thereafter (see Fig. 3 (b)). This predicts that PtAsP would exhibit good thermal stability. The entropy (Fig. 3(c)), which doesn't follow linearly with temperature, has a value of $276.83 \mathrm{~J} / \mathrm{K} /(\mathrm{N} \mathrm{mol})$ at $300 \mathrm{~K}$. The heat capacity at constant volume $\left(C_{\mathrm{v}}\right)$, for PtAsP under the harmonic approximation of the Debye model is shown in Fig. 3 (d). PtAsP had a $C_{\mathrm{v}}$ value of $259.55 \mathrm{~J} / \mathrm{K} /(\mathrm{N}$ mol $)$ at $300 \mathrm{~K}$ and 292.09 J/K/(N mol) at $730 \mathrm{~K}$, which is the point at which the Dulong and Petit's rule (the point at which $C_{\mathrm{v}}$ almost become a constant at high temperature) was obeyed.
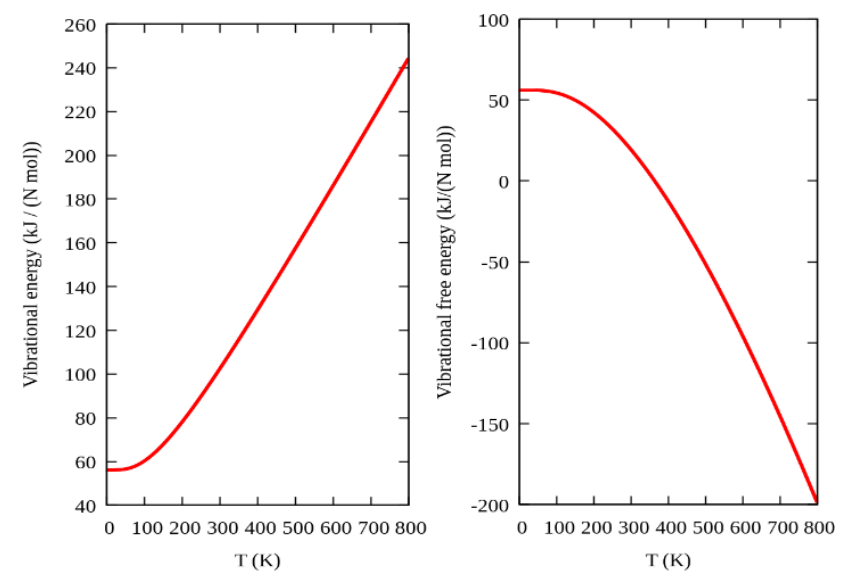

(a)

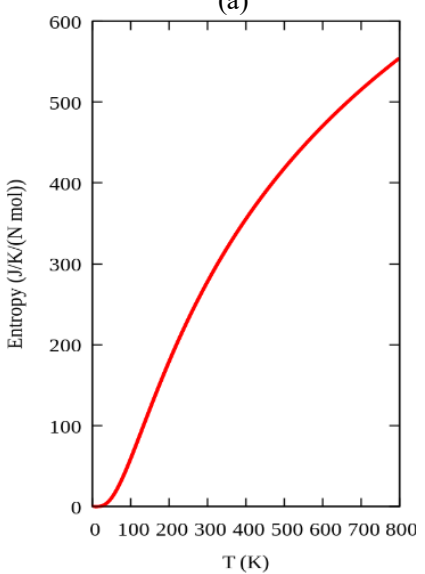

(c)

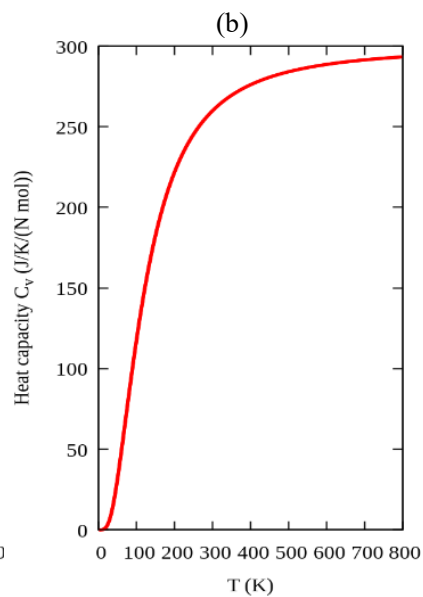

(d)

Fig. 3. Calculated vibration energy (a), vibration free energy (b), entropy (c) and constant volume heat capacity (d) for PtAsP.

\section{Transport Properties}

According to the Fermi-Dirac distribution function $f(E)$, the probability of finding a quantum system with energy $E$ is governed by $f(E)=1 /(1+\exp ((E-\varepsilon) / k T))$. $\varepsilon$ is the chemical potential of the system. The chemical potential $\varepsilon$, is a thermodynamic function which can be defined as the energy that a specie can absorb or release due to a change in the number of the specie in a given particle. The position of $\varepsilon$ plays an important role on the electronic structure and therefore the general material properties. In materials 
science and engineering, element substitution or doping is basically used to manipulate the chemical potential. In semiconductors, $\varepsilon=0$ at the top of the valence band [20]. The total number of carriers and temperature are used to determine the chemical potential. Thus, the variation of $\varepsilon$ with varying temperature and doping is desirable when investigating transport/thermoelectric properties. Shown in Fig. 4 (a) is the calculated variation of chemical potential with respect to temperature, where $\varepsilon$ has a slight variation with temperature for PtAsP. At $300 \mathrm{~K}$ and at $\varepsilon=0$, the Seebeck coefficient (see Fig. 4(a)) is positive $(S>0)$, hence, PtAsP is predicted to be a $p$-type material. The Seebeck coefficient $(S)$ was found to decrease with increasing temperature from $1060 \mu \mathrm{V} / \mathrm{K}$ (at $300 \mathrm{~K}$ ) to $444 \mu \mathrm{V} / \mathrm{K}$ (at $800 \mathrm{~K})$. The electrical conductivity $(\sigma / \tau)$ is almost independent of temperature (see Fig. 4 (b)). Its maximum electrical conductivity occurs at $\mu=0.09 \mathrm{Ry}$. It is however evident from Fig 4(b), that the electrical conductivity is higher for the $n$-type PtAsP than its $p$-type. The electronic thermal conductivity $(\kappa / \tau$, Fig. 4 (c)) increases with temperature. It increased from $20.67 \times 10^{14} \mathrm{~W} / \mathrm{mKs}$ (at $300 \mathrm{~K}$ ) to $47.95 \times 10^{14} \mathrm{~W} / \mathrm{mKs}$ (at $800 \mathrm{~K}$ ). The plot of the power factor $\left(\mathrm{S}^{2} \sigma\right.$, Fig. 4 (d)) shows that it increases with temperature for both the $n$-type and $p$-type PtAsP. However, the power factor was higher for the $n$-type material than the $p$-type PtAsP. At lower temperature, it can be seen that the $p$-type would exhibit higher power factor than the $n$-type PtAsP.

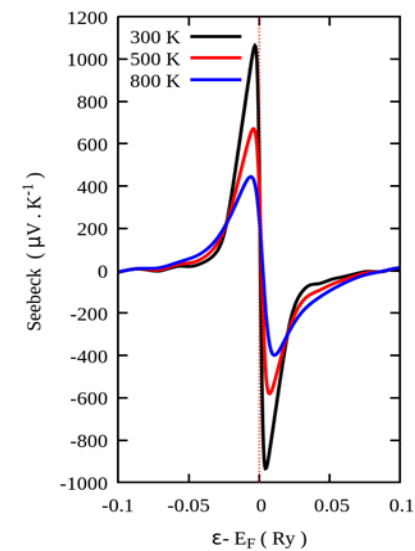

(a)

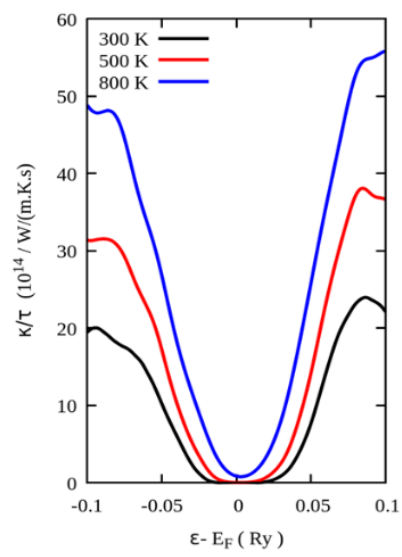

(c)

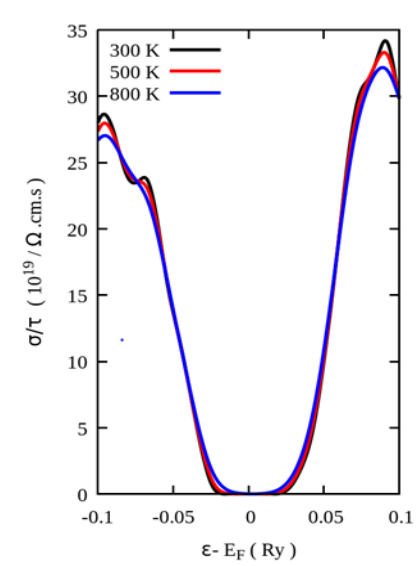

(b)

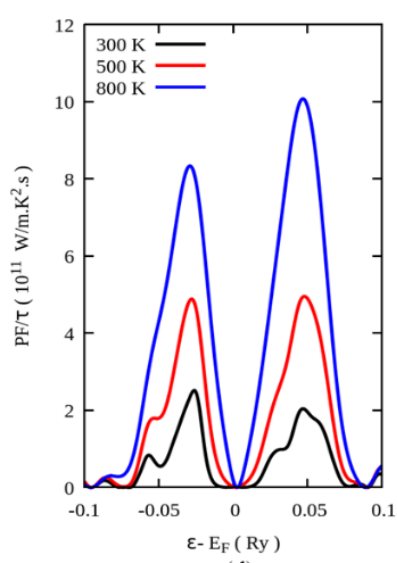

(d)
Fig. 4. (a) Variation of Seebeck coefficient with chemical potential. (b) variation of electrical conductivity with chemical potential. (c) variation of electronic thermal conductivity with chemical potential. (d) variation of power factor with chemical potential.
Attention is turned next to the variation of transport properties with doping and temperature. The transport properties were evaluated for doping concentrations of $10^{16} \mathrm{~cm}^{-3}, 10^{17} \mathrm{~cm}^{-3}, 10^{18} \mathrm{~cm}^{-3}$ and $10^{19} \mathrm{~cm}^{-3}$. The Seebeck coefficient (Fig. 5(a)) is almost constant with all doping concentrations and it increases with increase in temperature. It can also be observed that the concentration of doping on the electronic thermal conductivity (Fig. 5 (c)) and the power factor (Fig. 5 (d)), is also almost indistinguishable. The two parameters are found to increase with temperature. The electrical conductivity on the other hand, was found to be marginally improved with hole concentration of $10^{16} \mathrm{~cm}^{-3}, 10^{17} \mathrm{~cm}^{-3}$ and $10^{18} \mathrm{~cm}^{-3}$ above that of $10^{19} \mathrm{~cm}^{-3}$. The electrical conductivity was found to decline with increase in temperature. This is a trend that could be expected because electrical conductivity depends directly upon carrier concentrations and mobility. Moreover, the mobility is inversely related to temperature, and thus the electrical conductivity.

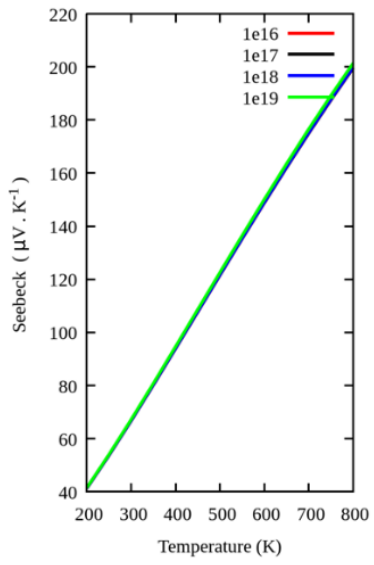

(a)

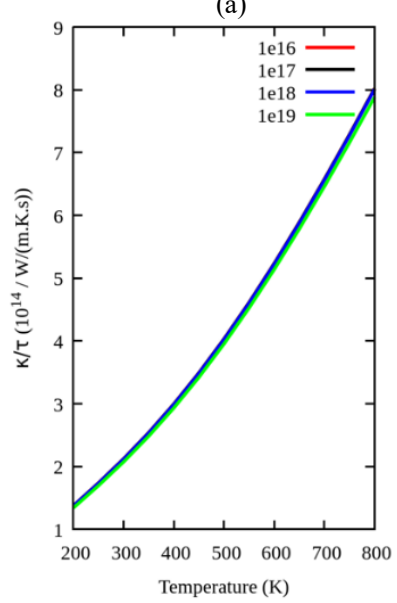

(c)

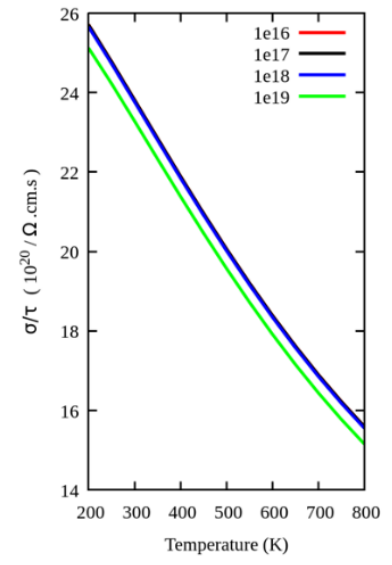

(b)

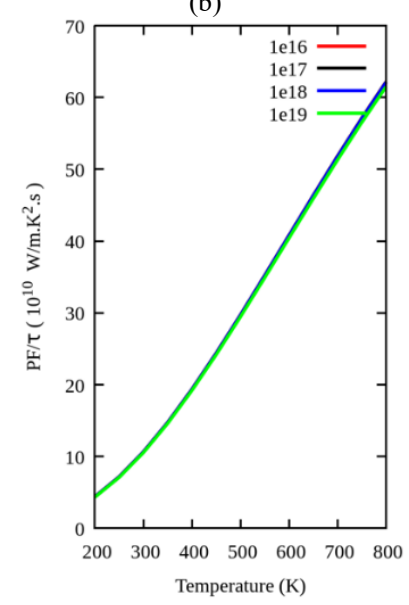

(d)
Fig. 5. (a) Seebeck coefficient, (b) electrical conductivity, (c) electronic thermal conductivity, and (d) power factor with respect to temperature at various hole doping concentrations.

\section{CONCLUSION}

First-principles calculation methods have been used to investigate the electronic structure, elastic, dynamics as well as the thermoelectric properties of PtAsP mixed cubic pyrite phase. Despite the longtime experimental discovery of this compound, almost nothing is known about the investigated properties. The results obtained in this study 
are good enough to warrant the exploitation of PtAsP cubic pyrite for technological applications. Moreover, the merit of this work is not only in evaluating the various discussed data, but also explaining their meanings in real material usage terms. Therefore, the various results are expected to fill obvious data gaps while at the same time inspiring additional future works.

\section{REFERENCES}

[1] Hung, A., Muscat, J., Yarovsky, I., \& Russo, S.P. (2002). Densityfunctional theory studies of pyrite $\mathrm{FeS}_{2}(100)$ and (110) surfaces. Surface Science 513(3), 511 - 524.

[2] Mori, K., Usui, H., Sakakibara, H., \& Kuroki, K. (2014). Theoretical expectation of large Seebeck effect in $\mathrm{PtAs}_{2}$ and $\mathrm{PtP}_{2}$. Journal of the Physical Society of Japan, 83(2), 023706.

[3] Hicks, D., Mehl, M. J., Gossett, E., Toher, C., Levy, O., Hanson, R. M., Hart, G. L. W., \& Curtarolo, S. (2019). The AFLOW Library of Crystallographic Prototypes: Part 2, Computational Materials Science, 161, S1-S1011 (doi=10.1016/j.commatsci.2018.10.043).

[4] Hulliger, F. (1963). Electrical properties of pyrite-type and related compounds with zero spin moment. Nature, 200, pp. 1064 -1065.

[5] de Jong M, Chen W, Notestine R, Persson K, Ceder G, Jain A, Asta M, Gamst A. A Statistical Learning Framework for Materials Science: Application to Elastic Moduli of k-nary Inorganic Polycrystalline Compounds. Scientific Reports 6: 34256 (2016) | doi:10.1038/srep34256.

[6] Giannozzi, P., Baroni, S., Bonini, N., Calandra, M., Car, R., Cavazzoni, C., Ceresoli, D., Chiarotti, G.L., Cococcioni, M., Dabo, I., Dal Corso, A., Fabris, S., Fratesi, G., De Gironcoli, S., Gebauer, R., Gerstmann, U., Gougoussis, C., Kokalj, A., Lazzeri, M., MartinSamos, L., Marzari, N., Mauri, F., Mazzarello, R., Paolini, S., Pasquarello, A., Paulatto, L., Sbraccia, C., Scandolo, S., Sclauzero, G., Seitsonen, A. P., Smogunov, A., Umari, P., \& Wentzcovitch, R.M. (2009). QUANTUM ESPRESSO: a modular and open-source software project for quantum simulations of materials. Journal of Physics: Condensed Matter, 21(39), 21395502.

[7] Perdew, J.P., Burke, K., \& Ernzerhof, M. (1996). Generalized gradient approximation made simple. Physical Review Letters, 77 (18), 3865-3868.

[8] H. J. Monkhorst and J. D. Pack, Phys. Rev. B13, 5188 (1976).

[9] F.D. Murnaghan, Proc. Natl. Acad. Sci. U. S. A. 30, 244 (1944).

[10] Baroni, S., De Gironcoli, S., Dal Corso, A., \& Giannozzi, P. (2001). Phonons and related crystal properties from density-functional perturbation theory. Reviews of Modern Physics, 73, 515-562.

[11] K.H. Madsen and J. Singh Comput. Phys. Commun., 175, 67 (2006).

[12] Hill, R. (1952). The elastic behavior of a crystalline aggregate. Proceedings of the Physical Society, 65, 349 - 354.

[13] Born, M. (1940). On the stability of crystal lattices. Mathematical Proceedings of the Cambridge Philosophical Society, 36, 160-172.

[14] Bensalem S, Chegaar M, Maouche D and Bouhemadou A. Theoretical study of structural, elastic and thermodynamic properties of CZTX $(\mathrm{X}=\mathrm{S}$ and Se) alloys. Journal of Alloys and Compounds. 2014; 589:137-142. http://dx.doi.org/10.1016/j. jallcom.2013.11.113.

[15] Guemou M, Abdiche A, Riane R and Khenata R. Ab initio study of the structural, electronic and optical properties of BAs and $\mathrm{BN}$ compounds and BNxAs1-x alloys. Physica B: Condensed Matter. 2014; 436:33-40. http://dx.doi.org/10.1016/j. physb.2013.11.030.

[16] Gao X, Jiang Y, Zhou R and Feng J. Stability and elastic properties of $\mathrm{Y}-\mathrm{C}$ binary compounds investigated by first principles calculations. Journal of Alloys and Compounds. 2014; 587:819-826. http://dx.doi.org/10.1016/j.jallcom.2013.11.005.

[17] Zhang M, Yan H, Zhao Y and Wei Q. Mechanical properties and atomistic deformation mechanism of spinel-type BeP2N4. Computational Materials Science. 2014; 83:457-462. http:// dx.doi.org/10.1016/j.commatsci.2013.11.044.

[18] Wang S, Li JX, Du YL and Cui C. First-principles study on structural, electronic and elastic properties of graphenelike hexagonal Ti2C monolayer. Computational Materials Science. 2014; 83:290-293. http://dx.doi.org/10.1016/j. commatsci.2013.11.025.

[19] Feng LP, Li N, Yang MH and Liu ZT. Effect of pressure on elastic, mechanical and electronic properties of WSe2: a firstprinciples study. Materials Research Bulletin. 2014; 50:503508. http://dx.doi.org/10.1016/j.materresbull.2013.11.016.

[20] Bensalem S, Chegaar M, Maouche D and Bouhemadou A. Theoretical study of structural, elastic and thermodynamic properties of CZTX (X $=\mathrm{S}$ and Se) alloys. Journal of Alloys and Compounds. 2014; 589:137-142. http://dx.doi.org/10.1016/j. jallcom.2013.11.113.

[21] Kanchana V and Ram S. Electronic structure and mechanical properties of $\mathrm{Sc} 3 \mathrm{AC}(\mathrm{A}=\mathrm{Al}, \mathrm{Ga}, \mathrm{In}, \mathrm{Tl})$ and $\mathrm{Sc} 3 \mathrm{BN}(\mathrm{B}=\mathrm{Al}, \mathrm{In}): \mathrm{Ab}-$ initio study. Intermetallics. 2012; 23:39-48. http:// dx.doi.org/10.1016/j.intermet.2011.12.014.

[22] Greaves GN, Greer AL, Lakes RS and Rouxel T. Poisson's ratio and modern materials. Nature Materials. 2011; 10:823-837. PMid:22020006. http://dx.doi.org/10.1038/nmat3134.

[23] Güler $M$ and Güler E. Embedded atom method-based geometry optimization aspects of body-centered cubic metals. Chinese Physics Letters. 2013; 30(5):056201. http://dx.doi. org/10.1088/0256307X/30/5/056201.

[24] Tariq, S., Ahmed, A., Saad, S., \& Tariq, S. (2015). Structural, electronic and elastic properties of the cubic CaTiO under pressure: A DFT study. AIP Advances, 5 (7), 077111.

[25] Ustundag M, Aslan M and Yalcin BG. The first-principles study on physical properties and phase stability of Boron-V (BN, BP, BAs, $\mathrm{BSb}$ and $\mathrm{BBi}$ ) compounds. Computational Materials Science. 2014; 81:471-477. http://dx.doi.org/10.1016/j. commatsci.2013.08.056.

[26] Güler, E., \& Güler, M. (2014). Phase transition and elasticity of gallium arsenide under pressure. Materials Research, 17(5), 12681272 .

A. I. Popoola is a faculty member at the Federal University of Technology, Akure, Ondo State, Nigeria. He is currently an Associate Professor and a member of the university senate. His research centers on electronics and computational materials modeling.

B. S. Agboola is a graduate student under the advice of AIP. His research work focusses on optimization of battery materials. He also teaches physics at the Continuing Education Center of the Federal University of Technology, Akure, Ondo State, Nigeria. 